\title{
Natura@economía
}

ISSN 2226-9479 (Versión electrónica)

Website: http://revistas.lamolina.edu.pe/index.php/neu

\section{Comercialización de miel de abeja del bosque seco, distrito de Motupe,} Lambayeque, Perú

\section{Commercialization of honey from dry forest, Motupe district, Lambayeque, Peru}

\author{
Waldemar Mercado ${ }^{1}$; Diana Rimac ${ }^{2}$ \\ ${ }^{1}$ Universidad Nacional Agraria La Molina, Facultad de Economía y Planificación, Apartado postal 12-056 - La \\ Molina, /Lima, Perú. Email: wmercado@lamolina.edu.pe \\ ${ }^{2}$ Universidad Nacional Agraria La Molina, Facultad de Economía y Planificación, Lima, Perú. Email:drimac. \\ reyes@gmail.com
}

Recibido: 25 abril del 2019; Aceptado: 20 junio del 2019

\begin{abstract}
Resumen
El objetivo del estudio fue analizar la comercialización, competitividad y rentabilidad de la miel de abeja proveniente del bosque seco, así como verificar las preferencias de los consumidores urbanos en la compra del producto. Los métodos de colecta de información primaria fueron entrevistas a agentes de la cadena productiva, encuestas, grupos focales y talleres con productores de Tongorrape, y encuestas a consumidores de Chiclayo y Lima, lo que permitió reconocer a los diversos agentes y canales de comercialización, determinar costos de producción, estimar márgenes brutos y evaluar beneficios netos. Los resultados evidencian que la miel orgánica elaborada por productores organizados es rentable y se distribuye a nivel nacional, en tanto, la miel convencional que recae en productores individuales, depende de los acopiadores, del canal de comercio y los precios, siendo necesario mejorar su gestión productiva y comercial. En conclusión, en la miel de abeja convencional predomina el comercio centralizado mientras en la miel orgánica prevalece su distribución vía canales directos y circuitos cortos, así como, existen diferencias en las preferencias de los consumidores de miel de abeja de las ciudades de Chiclayo y Lima.
\end{abstract}

Palabras clave: Comercio interno; comercialización; beneficio-costo; miel de abeja.

\begin{abstract}
The This study analyzes honey bee from the dry forest's marketing, competitiveness and profitability and verifies the consumer's preferences in the purchase of the product. Primary data collection methods were interviews to members of the productive chain, focus group and workshops with producers from Tongorrape and surveys to Chiclayo and Lima's consumers. The purposes of these methods were to recognize different agents and marketing channels and determine production costs. The results show that the organic honey produced by organized producers is profitable and it is distributed nationally; however, the conventional honey produced by not organized producers needs news marketing's channel. Finally, the conventional honey is distributed by direct channels and short circuits. In the same way, there are differences about preferences of honey bee's consume between Chiclayo and Lima cities. Keywords: Domestic trade; commercialization; profits; benefit-cost; honey bee.
\end{abstract}

Forma de citar el artículo: Mercado, W. \& Rimac, D. 2019. Comercialización de miel de abeja del bosque seco, distrito de Motupe, Lambayeque, Perú. Natura@economía 4(1): 24-37 (2019). 


\section{Introducción}

La biodiversidad forestal sustenta una amplia gama de bienes y servicios necesarios, y además proveen Servicios Ecosistémicos que impactan en el bienestar humano en forma directa o indirecta (Fisher et al., 2009; Costanza et al., 1997; Rodríguez et al., 2016). MEA (2005) clasificó los servicios ecosistémicos sobre la base de sus funciones en: (i) servicios de aprovisionamiento de productos; (ii) servicios de regulación de procesos ecológicos como el agua y el clima; (iii) servicios culturales de beneficios no materiales de actividad recreacional y valor espiritual; (iv) servicios de apoyo, que De Groot et al. (2010) clasifican como servicios de hábitat (de crianza, patrimonio genético, etc.).

El Perú cuenta con más de 73 millones de hectáreas (ha) de bosques, pero la deforestación erosiona esos recursos, así entre los años 2010 y 2013, se ha perdido en promedio $113 \mathrm{mil}$ ha de bosque al año, debido principalmente a la conversión de bosques en tierras agrícolas (SERFOR, 2015). En los bosques secos de la costa norte el cambio de uso de suelo lleva a la pérdida de recursos y biodiversidad (Sabogal, 2011), afectando negativamente a las comunidades, particularmente en Lambayeque, donde la estabilidad económica depende en parte de los recursos forestales, pues la economía agraria es soporte económico y de subsistencia para más de 400 mil familias campesinas (Beltrán, 2013), así se reportan pérdidas de 20 mil ha de bosque entre Olmos, Motupe, Salas, Jayanca, Pítipo, Íllimo y Mórrope (Cuentas, 2015).

Analizar las propiedades de los ecosistemas, derivadas de los servicios que ofrecen y de su valor de mercado ayuda a la toma de decisiones sobre el uso de la tierra, que deberían considerar su valor natural, cultural y económico (comercial y no-comercial) de bienes y servicios ecosistemicos, y la distribución espacial de esos valores, por lo que las estrategias de gestión forestal para su conservación se deben integrar al desarrollo territorial (Rodríguez et al., 2016; Cuentas, 2015), como herramienta para la formulación de políticas nacionales (SERFOR, 2015).

Los servicios ecosistémicos de provisión del bosque seco tropical son biodiversidad, plantas medicinales, ornamentales, néctar para apicultura, alimentos vegetales, madera, leña, entre otros (UICN, 2015). El bosque seco de Motupe ofrece diversidad multifloral para producir miel de abeja, que permite generar ingresos adicionales a la población rural y simultáneamente favorecer la conservación de la biodiversidad.

Según Philippe (1990) el Perú cuenta con razas de abejas carniola para apicultura de clima frío y la italiana para zonas cálidas, pero la mayoría son abejas "criollas" de la cruza no controlada de la abeja negra española, la italiana y más recientemente la africana (Braunstein, 2006). Indacochea et al. (2001) señala que el año 1999 se contaba con 100 mil colmenas, en tanto, el IV Censo Nacional Agropecuario (CENAGRO, 2012) muestra $40 \quad 082$ unidades agropecuarias (U.A.) con colmenas y 214276 colmenas en producción, destacando Cuzco (17 909 en total), Junín (15 309) y Cajamarca (11 841), Lambayeque ocupa la 13va posición con 4 221 colmenas en 806 U. A.

La comercialización de miel de abeja ocurre en el sector formal (supermercados, tiendas naturistas y bodegas) que presentan gran variedad de marcas procedentes de diferentes zonas del país, y el sector informal (mercados de abastos, ventas directas de productores, ferias, etc.), que concentra alrededor del $40 \%$ al $45 \%$ del volumen comercializado, pero como no es controlada, es difícil sistematizar la información de producción y comercio. Por ello, el objetivo del estudio fue analizar la comercialización, competitividad y rentabilidad de la miel de abeja proveniente del bosque seco de Tongorrape (distrito de Motupe), así como verificar las preferencias de los consumidores urbanos en la compra del producto.

\section{Materiales y métodos}

El estudio se llevó a cabo en Tongorrape, distrito de Motupe, provincia y departamento de Lambayeque. Motupe, es el principal distrito frutícola de la zona, se ubica a $79 \mathrm{~km}$ al norte de la ciudad de Chiclayo, siendo un nodo intermedio que articula la producción de los distritos vecinos a los mercados, por su territorio discurren los ríos Chotoque (norte) y Motupe (sur), limita al norte y oeste con el distrito de Olmos, al sur con Jayanca, al este 
con Chochope y Salas (Figura 1), siendo un distrito frutícola rodeado de bosques secos: (i) Bosques ribereños semi densos donde predominan el algarrobo y faique; (ii) Bosques ralos de pampas y sabanas donde predomina algarrobo y sapote; (iii) Bosques tipo matorral que predomina el algarrobo, palo verde, sapote en zonas de transición entre llanura y colinas; (iv) Bosques de colina y chaparrales donde predomina el hualtaco, palo santo, charan, algarrobo $\mathrm{y}$ arbustos (Yachi, 2014).

Las economías rurales son dependientes de la producción de frutos que se desarrollan en el valle, la pecuaria extensiva y provisión del bosque seco, en una superficie de 37,2 mil ha, de las cuales el $22 \%$ son terrenos agrícola bajo riego y $78 \%$ son bosques, montes y pastos, en secano, de uso ganadero, apícola y forestal. Las unidades agrarias ocupan alrededor de 50 mil ha del distrito, y son constituidos por: (i) Dos comunidades campesinas, San Julián con 1544 comuneros en $372 \mathrm{~km}^{2}$ y Tongorrape con 727 comuneros en de $121 \mathrm{~km}^{2}$ (Castañeda, 2016) cuyo territorio ocupa $71 \%$ de la superficie (CENAGRO, 2012); (ii) 1200 unidades agrarias menores de 10 ha, que ocupan $11 \%$ de la superficie; (iii) 313 medianas unidades, con áreas entre 10 a 20 ha, ocupan el $8 \%$ del total; (iv) 120 propiedades mayores de 20 ha ocupan el $9 \%$ de superficie. La propiedad familiar constituye fuente de autoempleo y se aprovecha, también, los productos del bosque como algarroba, madera, pasto, miel, algarrobina $\mathrm{y}$ cultivos temporales.
Los bosques secos son campos abiertos de propiedad comunal con densidad de 100 a 150 plantas/ha (INRENA, 2001).

La Comunidad Campesina de Tongorrape cuenta con 727 comuneros inscritos, tiene una extensión de $557,4 \mathrm{~km}^{2}$ y densidad poblacional de $43,1 \mathrm{hab} . / \mathrm{km}^{2}$ (Yachi, 2014), desarrolla cultivos de maíz, menestras y frutales. También se aprovecha el bosque seco, en forma individual o por la asociación Asprobos (Asociación de Protección de los Bosques Secos), con la crianza de animales, producción de miel y derivados. Asprobos, es fomentado por el Proyecto Algarrobo INRENA con algunas ONG (CICAP y CES Solidaridad).

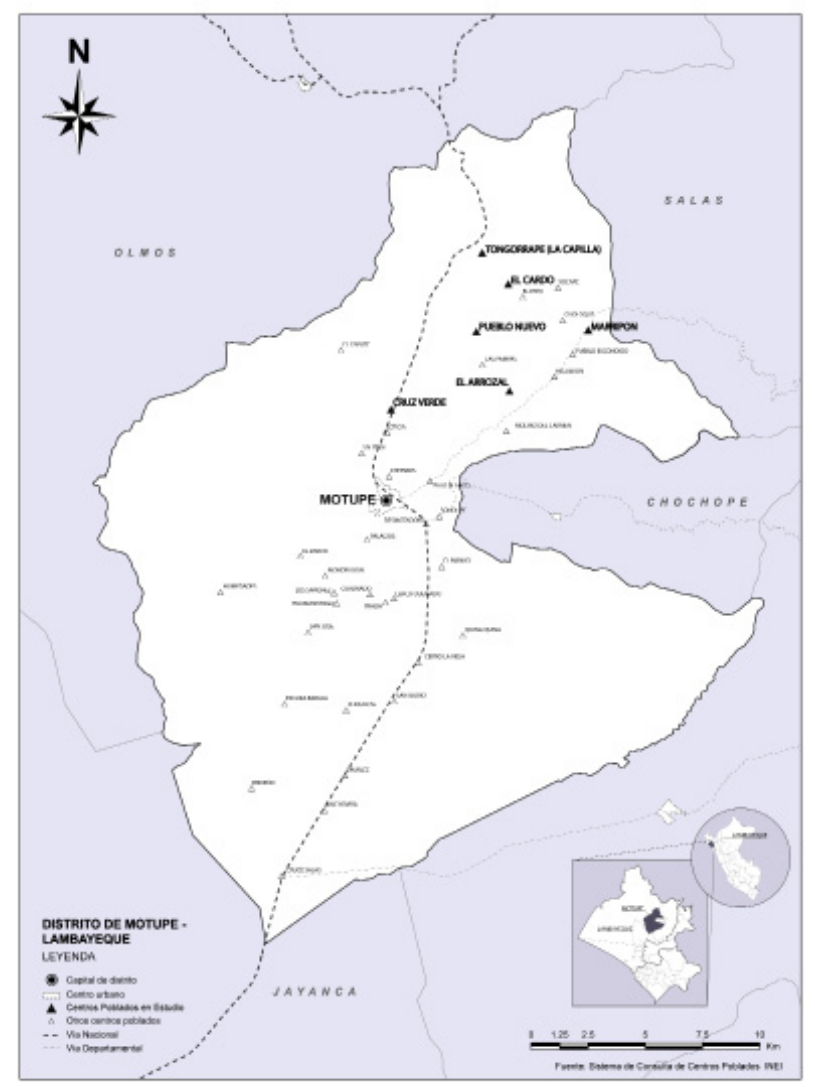

Figura 1: Mapa de ubicación del distrito de Motupe y centros poblados en estudio 
Los datos secundarios se obtuvieron del CENAGRO (2012), la Municipalidad de Motupe y el Instituto Nacional de Estadística e Informática (INEI, 2016). La información primaria se recopiló entre abril a junio 2015, considerando: (i) entrevistas a actores de Tongorrape (Alcalde, Presidente de ASPROBOS, productores e intermediarios comerciales); (ii) censo con encuestas a 39 productores apícolas en Tongorrape en sectores de La Capilla (8 encuestas), Cruz Verde (1), El Cardo (20), Arrozal (5), Marripón (4) y Pueblo Nuevo (1) ${ }^{1}$, y dos talleres participativos con productores; (iii) 150 encuestas a consumidores en tres distritos de la provincia de Chiclayo por niveles socioeconómicos ${ }^{2} \mathrm{~A} / \mathrm{B}$ representados por Chiclayo con 59858 familias y 79 encuestas, José Leonardo Ortiz con 36978 familias y 49 encuestas, y en nivel $\mathrm{C}$ por $\mathrm{La}$ Victoria con 17163 familias y 22 encuestas; (iv) 150 encuestas a consumidores en cinco distritos de Lima Metropolitana de niveles socio-económicos $\mathrm{A}, \mathrm{B}$ y $\mathrm{C}^{3}$, donde se realizan semanalmente ferias ecológicas, en San Isidro con 12845 familias (INEI, 2007) se aplicaron 16 encuestas, en Miraflores con 18821 familias se tuvo 23 encuestas, Surquillo con 19754 familias 24 encuestas, Barranco de 7501 familias nueve encuestas y en Santiago de Surco con 64076 familias, 78 encuestas.

Las muestras fueron obtenidas a partir del número de familias y se encuestó a la persona que decide el consumo familiar. Los cinco distritos de Lima componen 123 mil familias de cinco miembros y los tres distritos de Chiclayo 114 mil familias con cuatro miembros. El tamaño de la muestra se calculó con la fórmula de poblaciones finitas:

$$
\mathbf{n}=\frac{\mathrm{Z}^{2} \cdot \mathbf{p} \cdot \mathbf{q} \cdot \mathrm{N}}{\mathrm{E}^{2}(\mathrm{~N}-\mathbf{1})+\mathrm{Z}^{2} \cdot \mathbf{p} \cdot \mathbf{q}}
$$

\footnotetext{
El número de productores apícolas para cada sector son de registros de la Asociación ASPROBOS, a cargo número de productores inmersos en ella.

${ }^{2}$ El nivel socioeconómico se define en función a un grupo de variables (ocupación, educación, tamaño de la familia e ingresos), a partir de estudios realizados APEIM e IPSOS PERÚ (2014) establecen cinco niveles socioeconómicos

${ }^{3}$ La provincia de Lima tiene en el nivel socioeconómico A 4, 9\% de hogares $(0,8 \%$ en A1 y $4,1 \%$ en A2), en NSE $\mathrm{B}$ el $18,8 \%$ de hogares $(7,7 \%$ en $\mathrm{B} 1$ y $11,1 \%$ en $\mathrm{B} 2)$, en NSE C el $40,8 \%$ de hogares $(25,1 \% \%$ en $\mathrm{C} 1$ y $15,6 \%$ en (APEIM, 2014).
}

$\mathrm{n}=$ muestra.

$Z=$ nivel de confianza al $95 \%$ el valor de $Z$ es 1,96 .

$\mathrm{p}=$ probabilidad de éxito de 0,5 , que indica la máxima varianza. $\mathrm{q}=(1-\mathrm{p})$ igual a 0,5 .

$\mathrm{N}=$ tamaño de la población

$\mathrm{E}=$ máximo error permisible, consideró el valor $6 \%$ (demanda).

La tipología de los apicultores clasifica al productor en grupos homogéneos con estadísticas descriptivas a partir del número de colmenas en producción, forma de organización, tipo de miel que producen y producción anual apícola.

El Margen bruto de comercialización (Precio del consumidor- precio del productor / precio del consumidor) mide la distribución del valor generado entre los participantes, expresado en unidades monetarias por unidad. El Margen neto de comercialización (MNC), es el valor total generado menos los costos involucrados por la actividad, se mide como porcentaje sobre el precio final que percibe la intermediación como beneficio neto, al deducir los costos de mercadeo.

$\mathrm{MNC}=[($ Margen bruto - Costos de mercadeo $)$ $/$ (Precio pagado por el consumidor)] $\times 100$ "

El procesamiento de los datos se desarrolló con el Excel 2010 y SPSS para tabular las encuestas de productores y consumidores, y obtener los distintos indicadores.

El análisis FODA expone el diagnóstico de la actividad apícola de factores internos con las fortalezas (F) y debilidades (D) que posee (matriz EFI), y de factores externos con las oportunidades (O) y amenazas (A) existentes (matriz EFE). En el taller participativo los productores asignaron calificación entre 1 y 4 para indicar si el factor representa una debilidad mayor (1) o menor (2), y una fortaleza menor (3) o mayor (4) (EFI), o una respuesta superior (4), mayor a la media (3), media (2) y mala respuesta (1) (EFE), posteriormente se asignó un peso de 0 (no importante) y 1 (muy importante) a cada factor y se multiplicó por su calificación para determinar su ponderación en cada variable, y se sumó esas calificaciones para determinar el total. En la matriz EFI un valor debajo de 2,5 señala debilidad interna, y mayor a 2,5 
indica fortaleza interna. En la matriz EFE un valor debajo de 2,5 señala que se enfrentan mayores amenazas y mayor a 2,5 indica que la realidad externa es favorable y presenta oportunidades.

La Matriz de estrategias permite diseñar estrategias que aprovechan las ventajas de las oportunidades, y las defensivas buscan disminuir el impacto de amenazas externas. Las estrategias adaptativas mejoran las debilidades internas y las de supervivencia reducen la posibilidad de caer en desbalance frente a las amenazas existentes. La zona de estudio presenta escasa información, por lo que fue crucial la colecta de información primaria.

\section{Resultados y discusión}

El CENAGRO (2012) permite establecer las superficies agrícolas y no agrícolas en el distrito de Motupe (Tabla 1), donde el bosque seco representa $60 \%$ del total de ha, lo cual demuestra que la apicultura podría convertirse en una actividad relevante para el ingreso de los pobladores. En el tamaño de U. A. el 85,2\% poseen menos de 9,9 ha, $13,3 \%$ tiene entre 10 a 50 ha, y $1,6 \%$ más de 50 ha (CENAGRO, 2012).

Del Censo a productores apícolas en los seis sectores de Tongorrape, consultados sobre el aporte a los ingresos familiares, el $51 \%$ considera a los cultivos más importante, el $45 \%$ la apicultura, el 2,8\% la ganadería y $1,2 \%$ la forestería. En promedio el productor dedica 2,75 ha a la ganadería y 2 ha para
La cadena productiva como sistema constituidoporactores interrelacionadosy por una sucesión de operaciones de producción, transformación y comercialización de un producto en un entorno determinado, donde intervienen actores directos que actúan e interactúan en los diversos eslabones de la cadena (en algún momento son propietarios del producto), y actores indirectos que brindan apoyo a los actores directos como proveedores de insumos o servicios.

En la cadena productiva de la miel de abeja existen 39 apicultores que en promedio poseen 7,5 colmenas, con un total aproximado de 285 colmenas. Se identifican $80 \%$ de pequeños apicultores (con rangos entre 5 a 15 colmenas por productor) y 20\% medianos (mayores a 40 colmenas por productor ${ }^{5}$ ). El nivel organizativo es de ASPROBOS conformado por 20 pequeños y 8 medianos productores, quienes desde el 2002 con ayuda del Programa de las Naciones Unidas para el Desarrollo (PNUD) lograron tener apoyo financiero para el manejo, conservación y protección del bosque seco, elaboran miel con certificación orgánica de Biolatina en estándar nacional y comercializan sus productos en las ferias ecológicas. La producción de miel en cosechas normales es $132 \mathrm{~kg} / \mathrm{año}$ promedio, y llega hasta $300 \mathrm{~kg} / \mathrm{año}$ durante el Fenómeno El Niño (FEN) cuando las precipitaciones fructifican el bosque. Los 11 apicultores independientes elaboran miel convencional y sin valor agregado para el consumo personal y/o venta minorista.

Tabla 1: Superficie agrícola y no agrícola del distrito de Motupe - Lambayeque

\begin{tabular}{lcccccccc}
\hline \multirow{2}{*}{$\begin{array}{l}\text { Distrito de } \\
\text { Motupe }\end{array}$} & Total & \multicolumn{4}{c}{ Superficie agrícola } & \multicolumn{4}{c}{ Superficie no agrícola } \\
\cline { 2 - 10 } & Total & $\begin{array}{c}\text { Bajo } \\
\text { riego }\end{array}$ & $\begin{array}{c}\text { En } \\
\text { secano }\end{array}$ & Total & $\begin{array}{c}\text { Pastos } \\
\text { naturales }\end{array}$ & $\begin{array}{c}\text { Montes y } \\
\text { bosques }\end{array}$ & $\begin{array}{c}\text { Otra clase } \\
\text { de tierras }\end{array}$ \\
\hline Superficie (ha) & 51338 & 8634 & 7910 & 724 & 42704 & 1342 & 30854 & 10509 \\
\hline Porcentaje & 100 & 16,8 & 15,4 & 1,4 & 83,2 & 2,6 & 60,1 & 20,5 \\
\hline
\end{tabular}

Fuente: Censo Nacional Agropecuario 2012 (desagregación por distritos)

forestería ${ }^{4}, 1,98$ ha para los cultivos, 0,57 ha para apicultura, no existe arrendamiento de tierras, el $73 \%$ de productores apícolas pertenecen a ASPROBOS y el $27 \%$ son pequeños productores independientes.

\footnotetext{
${ }^{4}$ Las actividades de ganadería, forestería y apicultura se realizan con uso de tierras ocupadas en el bosque seco, siendo que las parcelas compuestas por propiedades menores a 2 ha dedicadas a la producción agrícola.
}

La producción de miel de abeja, que es el principal producto, ocurre en los meses de mayo, junio y diciembre; una colmena produce $17,6 \mathrm{~kg} /$ año y $2,6 \%$ de colmenas están fuera de la localidad. La producción

\footnotetext{
${ }^{5}$ El rango entre 15 a 40 no se han considerado porque no se han encontrado datos sobre productores que trabajen con ese número de colmenas.
} 
total anual es $5019 \mathrm{~kg} / \mathrm{año,} \mathrm{también} \mathrm{generan}$ polen $(3 \mathrm{~kg} /$ colmena/año) y cera $(1,8 \mathrm{~kg} /$ colmena/año) pero la mayoría no identifica su valor de mercado, por lo que muchos panales al término de su vida útil son destruidos.

Los actores que proporcionan servicios son la Asociación peruana de pequeños productores ecológicos (APEPROECO) que comercializan sus productos en forma organizada. En certificaciones participan DIGESA, SENASA, INDECOPI y Biolatina. Los proveedores de insumos proporcionan materiales de producción y medicinas siendo el principal abastecedor "Miel Andina" de Chiclayo. El 89\% de productores no cuentan con capacitación y el $11 \%$ tienen asesoramiento con costo anual de $\mathrm{S} / 350$, otros recibieron capacitación de una ONG o la Universidad Agraria sin costo, no existe uso del financiamiento bancario, pero la asociación APEPROECO busca acceder a créditos por proyectos de alternativas sostenibles al bosque seco.

\section{Canales de comercialización para la miel de abeja}

La comercialización son actividades que llevan productos desde el punto de producción hasta los consumidores finales, y tiene tres componentes: la concentración del producto desde los productores hasta el mercado; la dispersión del bien desde el mayorista o procesador a minoristas y consumidores; y la igualación para emparejar las cantidades demandadas y ofrecidas en el tiempo (Martínez, 2005), también se refiere a los aspectos físicos del transporte, almacenaje, acondicionamiento y procesamiento (Coscia, 1978). En países en desarrollo, el pequeño productor posee limitada información de precios e intermediarios, formando procesos simples y con poco valor agregado en el mercadeo.

Los canales de comercialización han evolucionado como respuesta a cambios del entorno. En la mayoría de productos, los intermediarios participan en forma secuencial en el flujo físico del bien para llevarlo al mercado (Caldentey, 2004; Martínez, 2005), presentan límites geográficos (áreas de mercado), económicos (gestión de volumen) y humanos (capacidad de interactuar) (Stern y El-Ansary, 1999,
Caldentey y De Haro 2004), y el margen de comercialización representa los costos y riesgos del mercadeo, además de la retribución de los agentes (Mendoza, 1991).

El comercio de miel de abeja identifica cinco actores: productor, acopiador, mercado de Motupe, mercado central (de Chiclayo), las ferias ecológicas regionales y de Lima.

Los productores venden en conjunto 4 $837 \mathrm{~kg} /$ año. El precio del productor en época normal es $7 \mathrm{~S} / . / \mathrm{kg}$ en miel convencional y $25 \mathrm{~S} / . / \mathrm{kg}$ por miel orgánica, durante el FEN es 10 soles $/ \mathrm{kg}$ por miel convencional y hasta $30 \mathrm{~S} / . / \mathrm{kg}$ por miel orgánica. El productor de miel orgánica destina a ventas al por mayor (2 $629 \mathrm{~kg} / \mathrm{año}$ ), al por menor (2 208 $\mathrm{kg} / \mathrm{año})$ y al autoconsumo (182 kg/año con consumo per cápita $0,96 \mathrm{~kg} / \mathrm{año}$ ), estos alcanzan mercados directos e incrementan sus márgenes de ganancia participando en el comercio de circuitos cortos (con valor agregado) en forma directa o descentralizada, considerando hasta un intermediario para que el producto llege al consumidor final, independientemente de la distancia recorrida.

Los intermediarios son: (i) Acopiadores, en número de dos agentes que enlazan los productores de miel convencional con el mercado; (ii) El mercado de Motupe donde los pobladores locales lo adquieren para revenderlos en la ciudad de Chiclayo y/o Lima, y para consumo familiar; (iii) El mercado central en Chiclayo que ofrece variedad en precios y tipos de miel de abeja; (iv) Las ferias ecológicas de Lima en Miraflores, Surquillo y Surco que venden miel orgánica de Tongorrape y de otras partes del Perú; (v) Las ferias de Chiclayo que promocionan productos de bosques secos de la región.

La Tabla 2 y Figura 2 muestran los canales de comercialización para la miel de abeja de Tongorrape. En la miel convencional predomina el comercio centralizado $(70 \%$ del total $)$ y también se distribuye en forma directa en Chiclayo y Motupe. En miel orgánica, la asociación ASPROBOS comercializa en circuitos cortos (40\%) llegando en mayor porcentaje a ferias ecológicas de Lima (75\%), feria Regional de Chiclayo, Cajamarca y universidades de Chiclayo (Pedro Ruiz Gallo y Santo Toribio de Mogrovejo) en 25\%. 
El $15 \%$ de producción convencional es ofrecida en el "Mercado Viejo" de Motupe a $\mathrm{S} / 10 \mathrm{~kg}$ con venta del productor e intermediarios, a las tiendas en Chiclayo va $10 \%$ que son llevados por acopiadores locales y "Miel Andina" que compra y vende miel producida en Tongorrape, también se ofrecen en casas naturistas de Chiclayo donde existen diversas marcas y pesos provenientes de Olmos, Cajamarca, la selva y Tongorrape, además de juguerías que utilizan para jugos y/o ensaladas y adquieren la miel de abeja a precio de $\mathrm{S} / 10$ soles por $\mathrm{kg}$. por kg. y al incorporar esos costos ascienden a S/ 23,91 kg. El proceso de venta considera costo de transporte y representación de $\mathrm{S} /$ $2 \mathrm{~kg}$. El componente de mayor gasto son materiales y maquinarias que participan con $84,13 \%$ y el mínimo gasto es en la alimentación y/o medicinas (Tabla 3).

El costo es dividido en "previo a la certificación" y "posterior a la certificación" lo que permite mayor aproximación a los costos económicos, pues la certificación se financia con proyectos de cooperación. Las ganancias mayores se obtienen cuando los apicultores no asumen directamente

Tabla 2: Comparativo según canal y mercado para la distribución de miel de abeja producido en la Localidad de Tongorrape

\begin{tabular}{|c|c|c|c|c|c|c|}
\hline & & onvenc & & & el orgán & \\
\hline & & & $\%$ & & & $\%$ \\
\hline Según canal y tipo de & Directo 1 & Tipo 1 & 15 & Directo & Tipo 1 & 15 \\
\hline comercio & Directo & Tipo 2 & 15 & Directo & Tipo 2 & 25 \\
\hline & Indirecto & & 70 & Indirecto & & 60 \\
\hline & & & $\%$ & & & $\%$ \\
\hline Según el mercado de & & & 70 & & & 20 \\
\hline destino & Otr & ones & - & Otr & ones & 5 \\
\hline & & & 30 & & & 75 \\
\hline
\end{tabular}

Fuente: Grupo focal a productores apícolas de Tongorrape (mayo 2014) y Figura 2.

\section{Precios, costos y rentabilidad de la miel de abeja}

Según la encuesta, para los productores el precio se determina según el mercado $(84,6 \%)$, el tiempo y la calidad $(7,6 \%)$, la competencia $(3,8 \%)$, y la miel adulterada $(3,8 \%)$; ellos recibieron $7 \mathrm{~S} / / \mathrm{kg}$ en miel convencional y $25 \mathrm{~S} / / \mathrm{kg}$ en miel orgánica. Para los consumidores, la marca, procedencia y lugar de venta son considerados en su diferenciación, pues consideran que podría haber adulteraciones del producto.

Los costos de producción, se refieren a gastos incurridos por el abastecimiento de insumos productivos y servicios técnicos para obtener una cantidad de producción. Los costos difieren según tamaño del productor y tipo de miel, agrupándose en tres componentes: materiales y maquinarias, mano de obra, y alimentación y/o medicinas. Los costos de producción de miel orgánica del productor de ASPROBOS, que al año realizan tres cosechas promedio en cinco colmenas con una producción de $17,61 \mathrm{~kg} /$ colmena y venden entre 25 a $30 \mathrm{~kg}$. El costo unitario previo a la certificación es S/ 20,12 los costos de la certificación y cuando el producto es vendido en Lima, aun cuando para ello se deban asumir los gastos de transporte del bien y de representación en las ferias ecológicas de Lima (Tabla 3).

Los costos de producción de miel convencional para un apicultor que produce en 7,5 colmenas con $17,61 / \mathrm{kg}$ por colmena se muestran en la Tabla 4. El precio de venta por $\mathrm{kg}$ fluctúa entre 6,5 y $8,0 \mathrm{~S} / / \mathrm{kg}$, y el costo unitario es $7,23 \mathrm{~S} / / \mathrm{kg}$, manifestando pérdida unitaria al venderla a precio mínimo al acopiador local, pero muchas labores la realizan los propios productores y ellos no lo contabilizan como parte del costo de producción.

La comparación de los costos y precios (mínimos y máximos) manifiestan pérdida unitaria si el precio de venta es mínimo y una ganancia si la miel de abeja es vendida a precio máximo. Para estos pequeños productores individuales la actividad apícola podría generar otros resultados si llevaran a cabo un trabajo asociativo con los otros productores de miel para favorecer la rentabilidad económica. 


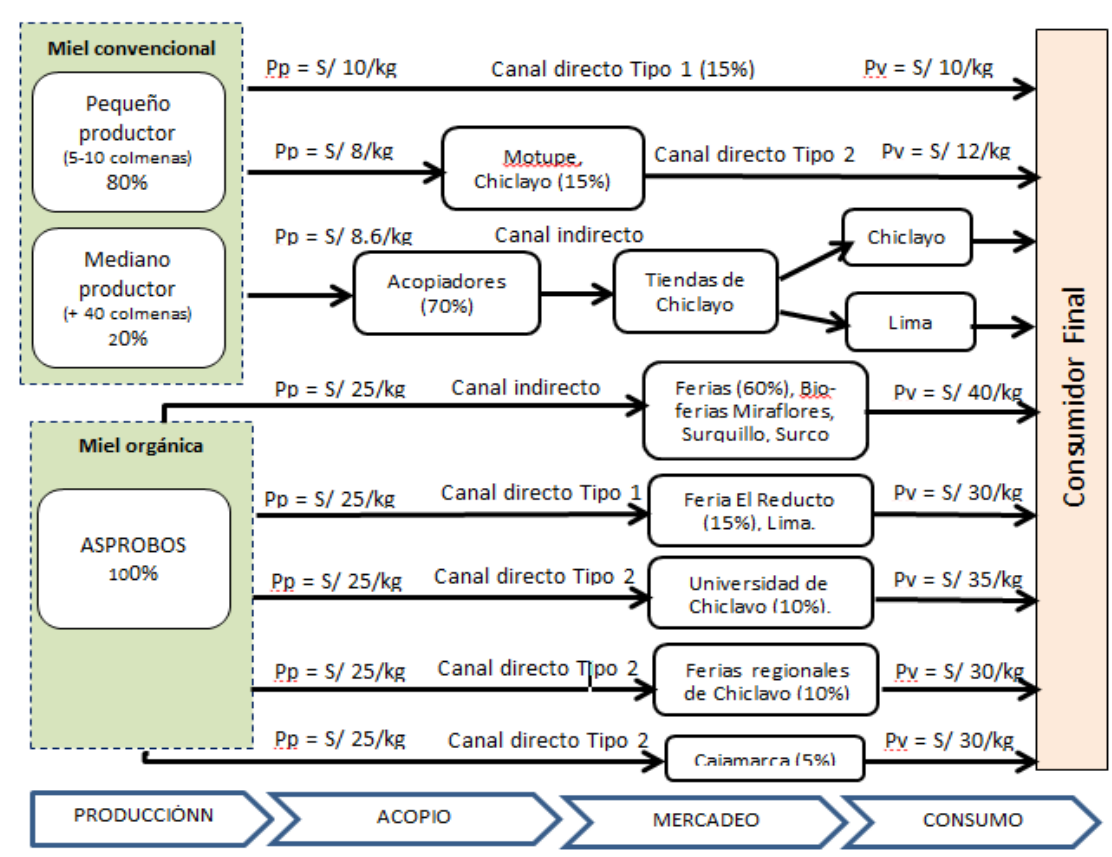

Figura 2: Canales de comercialización para la miel de abeja

Fuente: Focus group productores de Tongorrape (mayo 2015).

Tabla 3: Costo de producción promedio en miel orgánica y beneficios netos

\begin{tabular}{ccc}
\hline Componente & Cantidad $\begin{array}{c}\text { Por unidad } \\
\text { (S/) }\end{array}$ & $\begin{array}{c}\text { Costo de } \\
\text { producción }\end{array}$ \\
\hline Materiales y maquinaria: cajas, sahumador; baldes, espátula, & &
\end{tabular}

coladores, cuchara, jarra, trinche desopercu-lador, laminadora

(acero). Escobilla, mameluco, mascarilla, guantes, botas, colmenas, envases, manteles, centrifuga, comederos, cosecha, Diversos Diversos 4 440,8 decantador, panela orgánico $(25 \mathrm{~kg})$, olla desedificadora, balde de enfriamiento, hilo pabilo, etiqueta, precintos, carpa de cosecha, sogas, cera.

Jornales: revisión mensual dos personas (24 jornales), limpieza (dos veces/año), etiquetado y precinto (tres jornales).

Alimentación: bolsas de alimentos.

Costos de producción

Producción tres cosechas x cinco colmenas x 17,61 kg c/u Costo unitario kg. (previo a la certificación).

Costo de la certificación anual.

Costo de la certificación anual por kg producido

Costo de miel orgánica (por kg producido) . Simulaciones de beneficios netos

\begin{tabular}{|c|c|c|c|c|}
\hline \multicolumn{5}{|c|}{ Simulaciones de beneficios netos } \\
\hline Categorías de precios recibidos & $\begin{array}{l}\text { Mínimo } \\
\text { (1) }\end{array}$ & $\underset{(2)}{\text { Mínimo }}$ & $\begin{array}{l}\text { Máximo } \\
\text { (3) }\end{array}$ & Máximo \\
\hline $\begin{array}{r}\text { Precio kg } \\
\text { Ganancia unitaria }\end{array}$ & $\begin{array}{c}25,00 \\
4,88\end{array}$ & $\begin{array}{c}25,00 \\
1,09\end{array}$ & $\begin{array}{c}30.00 \\
7.88\end{array}$ & $\begin{array}{c}30,00 \\
4,09\end{array}$ \\
\hline Ganancia anual & 1289,0 & 287,9 & $2,081.5$ & 1001,1 \\
\hline
\end{tabular}

(1) No considera costos de certificación.

(2) Considera costos de certificación.

(3) No considera costos de certificación pero si gastos de transportes y representación en Lima.

(4) Considera costos de certificación y gastos de transportes y representación en Lima. 


\section{Márgenes de comercialización}

Los costos de comercialización constituyen el valor de insumos usados para agregar utilidad al producto, estos varían dependiendo de la estructura de producción y la localización del consumidor, e incluyen costos de acarreo, selección y clasificación, carga y descarga, fletes y envases, mermas, entre otros (Alarcón y Ordinola, 2002).

El Margen Bruto de comercialización refleja el precio pagado por el consumidor sin involucrar ningún costo. La Tabla 5 presenta esos márgenes según canal de comercio y tipo de productor. Los canales 2 y 3 tienen precio $\mathrm{S} / 8$ y obtienen un MBC de 33,3\%, es decir, de cada sol que paga el consumidor 0,77 va al productor $\mathrm{y}$ 0,33 a los intermediarios. En el canal 4 los precios generan MBC de 45,8\% y 33,3\%. Los productores organizados tienen precios mínimos y máximos, y variación en el precio al consumidor según el destino de distribución, en los canales 6,8 y 9 presentan un MBC mínimo de $16,7 \%$, y los canales 5 y 7 manifiestan diferencias según precios recibidos. Por tanto, el MBC es mayor en el comercio de miel convencional de los productores independientes, respecto a la miel orgánica producida por ASPROBOS.

El Margen neto de comercialización (MNC) mide la relación existente con el precio pagado por el consumidor involucrando los costos incurridos por los participantes. El beneficio relativo del capital total invertido refleja el desempeño económico de la actividad realizada (Díaz Roque, 2013). La Tabla 6 muestra estos indicadores.

El beneficio/costo $(\mathrm{B} / \mathrm{C})$ para la miel convencional con precio mínimo de $\mathrm{S} /$ 6,5 es de 0,89 , indica que por cada sol invertido se pierde 0,11 centavos (pérdida de $-6,08 \%$ ), pero si vende al precio máximo de $\mathrm{S} / 8$, la relación $\mathrm{B} / \mathrm{C}$ es 1,11 con margen de ganancia de $6,41 \%$. La diferencia entre ambas situaciones depende de la época de producción, los precios recibidos, el canal de distribución utilizado y la demanda regional.

Para la miel de abeja orgánica el B/C que se obtiene a precio en origen ( $\mathrm{S} / 25)$ es de 1,24 , señala que por cada sol invertido se obtiene 0,24 centavos. La venta al precio de $\mathrm{S} / 30$ con simulaciones que involucran o no el costo de la certificación, y del transporte y la representación en Lima, generaría indicadores de B/C entre 1,16 y 1,49 (Tabla 6). Por tanto, la actividad apícola es rentable en la zona, si bien que los productores también realizan otras actividades como la producción agrícola, pecuaria y comercio.

$\mathrm{La}$ producción de miel de abeja convencional resultaría rentable para los productores apícolas, pero las ganancias dependen del ciclo de distribución y de considerar valores reales en el costeo. La producción apícola orgánica genera ganancias a nivel regional y nacional aun considerando costos de certificación y representación, lo que permite respaldar la inversión incurrida, retribuir mano de obra y costos de la comercialización.

Tabla 4: Costo de producción promedio por productor de miel convencional

\begin{tabular}{|c|c|c|c|}
\hline Componente & Cantidad & $\begin{array}{l}\text { Costo por } \\
\text { unidad }\end{array}$ & $\begin{array}{l}\text { Costo anual } \\
\text { producción }\end{array}$ \\
\hline $\begin{array}{l}\text { Materiales y maquinaria: marcos, acero laminado, cajones, } \\
\text { pisos (una por colmena), tapas, entretapas, rejillas, guantes, } \\
\text { mamelucos, mascarillas, botas, cajas, baldes, centrífuga, } \\
\text { botellas, palanca, sahumador, escobilla, palana, machete, } \\
\text { manteles, filtro. } \\
\text { Jornales: revisión tres veces por mes (tres jornales). } \\
\text { Medicinas: remedios. }\end{array}$ & $\begin{array}{c}\text { Diversos } \\
3 \\
1\end{array}$ & $\begin{array}{c}\text { Diversos } \\
25 \\
60\end{array}$ & $\begin{array}{c}820,0 \\
75,0 \\
60\end{array}$ \\
\hline Costos de producción & & & 955,0 \\
\hline $\begin{array}{r}\text { Producción promedio } 7,5 \text { colmenas x } 17,61 \mathrm{~kg} \mathrm{c} / \mathrm{u} \\
\text { Costo unitario } \mathrm{kg}\end{array}$ & & & $\begin{array}{c}132,07 \\
7,23\end{array}$ \\
\hline $\begin{array}{r}\text { Simulaciones de beneficios netos } \\
\text { Categorías de precios recibidos } \\
\text { Precio kg } \\
\text { Ganancia unitaria } \\
\text { Ganancia anual }\end{array}$ & & $\begin{array}{c}\text { Mínimo (1) } \\
6,50 \\
-0,73 \\
-96,41\end{array}$ & $\begin{array}{c}\text { Máximo (2) } \\
8,00 \\
0,76 \\
100,37\end{array}$ \\
\hline
\end{tabular}

(1) Precio mínimo dependiendo si lo vende en su propio predio al acopiador local.

(2) Precio si lo vende en el mercado de Motupe.

Enero - Junio 2019 
Tabla 5: Margen Bruto de Comercialización por canal de distribución

\begin{tabular}{|c|c|c|c|c|c|c|}
\hline \multirow{2}{*}{ Productores } & \multirow{2}{*}{ Canal } & \multicolumn{2}{|c|}{ Precio al productor $\mathrm{S} / . / \mathrm{kg}$} & \multirow{2}{*}{$\begin{array}{c}\text { Precio al } \\
\text { consumidor } \mathrm{S} / / \mathrm{kg}\end{array}$} & \multicolumn{2}{|c|}{ MBC (\%) } \\
\hline & & Mínimo & Máximo & & Mínimo & Máximo \\
\hline \multirow{4}{*}{ Independientes } & 1 & 10,0 & 10,0 & 10,0 & - & - \\
\hline & 2 & 8,0 & 8,0 & 12,0 & 33,3 & 33,3 \\
\hline & 3 & 8,0 & 8,0 & 12,0 & 33,3 & 33,3 \\
\hline & 4 & 6,5 & 8,0 & 12,0 & 45,8 & 33,3 \\
\hline \multirow{5}{*}{ ASPROBOS } & 5 & 25,0 & 30,0 & 40,0 & 37,5 & 25,0 \\
\hline & 6 & 25,0 & 30,0 & 30,0 & 16,7 & 0,0 \\
\hline & 7 & 25,0 & 30,0 & 35,0 & 28,6 & 14,3 \\
\hline & 8 & 25,0 & 30,0 & 30,0 & 16,7 & 0,0 \\
\hline & 9 & 25,0 & 30,0 & 30,0 & 16,7 & 0,0 \\
\hline
\end{tabular}

\section{EI análisis FODA}

El FODA muestra la matriz de factores internos (EFI), siendo las principales fortalezas identificadas por los productores apícolas de la zona F2 (52\%), F1 y F5 (39\%), y las debilidades más importantes D4 y D5 (19\%) y D2 (10\%) (Tabla 7$)$. El valor de 2,68 de la matriz EFI señala que existen ligeramente más fortalezas que debilidades.

La matriz de factores externos (EFE) señala como oportunidades más importantes $\mathrm{O} 2$ y $\mathrm{O} 4(43 \%), \mathrm{O} 3(32 \%)$ y entre las debilidades A2 (43\%), A4 (32\%), A1 (29\%) (Tabla 7). La ponderación de 3,36 refleja que existen oportunidades que pueden neutralizar las amenazas que afectan la actividad apícola.

En la matriz de tendencias el primer cuadrante de crecimiento y desarrollo representa $35 \%$ de interrelación entre fortalezas y oportunidades para alcanzar mercados objetivos con mejoras continuas a nivel productivo y comercial, lo que permite establecer estrategias para uso de recursos internos que posibiliten aprovechar las ventajas de oportunidades externas con bienes diferenciados como se presenta en la Tabla 8. El segundo cuadrante de adaptabilidad tiene $30,8 \%$, el tercer cuadrante de neutralización de amenazas $20 \%$ y el cuarto cuadrante de supervivencia de $13,8 \%$.

\section{El consumo de miel de abeja en Chiclayo y Lima Metropolitana}

La población encuestada tiene mayor preferencia al consumo de miel de abeja en Chiclayo (92\%) que en Lima (81\%). Los que no adquieren el bien en Chiclayo es por el factor económico en $31 \%$, porque es ausente en el lugar de compra $23 \%$, no le atrae el bien $23 \%$, porque puede estar adulterado $15 \%$ u otro motivo 8\%. En Lima las respuestas fueron porque no lo encuentran en el lugar de compra o tienen poca información (36\%), no le atrae el producto $(50 \%)$, porque tienen bajo consumo de azúcar $(11 \%)$, y desconfianza en la adulteración (4\%).

Entre los demandantes de Chiclayo, la frecuencia de consumo es diario en $38 \%$, semanal $39 \%$, quincenal $7 \%$, mensual $10 \%$ y mayor a un mes $5 \%$. En Lima la frecuencia es $8 \%$ semanal, $16 \%$ quincenal, $40 \%$ mensual y $36 \%$ otra periodicidad. El 81\% expresaron que la miel de abeja es un producto esencial en su alimentación.

Tabla 6: Beneficio/Costo y MNC según tipos de miel de abeja con precios mínimos y máximos

\begin{tabular}{lcccccc}
\hline Tipo miel de abeja Precio venta (S/) & Beneficio & Costo & Beneficio/costo & MNC (\%) & Mercado \\
\hline \multirow{2}{*}{ conven-cional } & Mín. 6,5 & 858,46 & 954,86 & 0,89 & $-6,08$ & Regional \\
\cline { 2 - 7 } & Máx. 8,0 & 1056,60 & 954,86 & 1,11 & 6,41 & Regional \\
\hline \multirow{3}{*}{ Miel orgánica } & \multirow{2}{*}{ Mín. 25,0 } & 6603,75 & $5314,70(1)$ & 1,24 & 16,26 & Regional \\
\cline { 2 - 7 } & & 6603,75 & $5843,00(2)$ & 1,13 & 9,60 & Nacional \\
\cline { 3 - 7 } & \multirow{3}{*}{ Máx. 30,0 } & 7924,15 & $5314,70(1)$ & 1,49 & 28,22 & Regional \\
\cline { 2 - 7 } & & 7924,15 & $5843,00(2)$ & 1,30 & 22,51 & Nacional \\
\cline { 2 - 7 } & & 7924,15 & $6315,82(3)$ & 1,25 & 17,40 & Regional \\
\cline { 2 - 7 } & & 7924,15 & $6844,13(4)$ & 1,16 & 11,69 & Nacional \\
\hline
\end{tabular}

(1) No incluye costos de certificación.

(2) Incluye costos de transporte y representación pero no de la certificación.

(3) Incluye costo de certificación.

(4) Incluye costos de certificación, transporte y representación. 
Tabla 7: Matriz de evaluación de factores internos "EFI" y factores externos "EFE"

\begin{tabular}{|c|c|c|c|}
\hline FACTORES CRÍTICOS & \multirow{2}{*}{ Peso } & \multirow{2}{*}{$\begin{array}{l}\text { Califica- } \\
\text { ción }\end{array}$} & \multirow{2}{*}{$\begin{array}{l}\text { Ponde- } \\
\text { ración }\end{array}$} \\
\hline FORTALEZAS & & & \\
\hline $\begin{array}{l}\text { F1 Personas con experiencia en la producción de miel de abeja y en el } \\
\text { mantenimiento de colmenares. }\end{array}$ & 0,10 & 4 & 0,39 \\
\hline F2 Producción ecológica sustentada mediante la certificación. & 0,13 & 4 & 0,52 \\
\hline $\begin{array}{l}\text { F3 Organizaciones establecidas para la producción apícola, con la } \\
\text { participación en el Consejo Regional de Producción Orgánica. }\end{array}$ & 0,06 & 3 & 0,19 \\
\hline $\begin{array}{l}\text { F4 Existe conciencia frente a los temas de la conservación y el manejo } \\
\text { sostenible del bosque seco. }\end{array}$ & 0,06 & 4 & 0,26 \\
\hline $\begin{array}{l}\text { F5 Instituciones externas a la región como APEPROECO, PROMPERU y } \\
\text { Cámara de Comercio que brindan apoyo a la actividad. }\end{array}$ & 0,10 & 4 & 0,39 \\
\hline F6 Producción de miel del bosque seco es identificado por el consumidor. & 0,06 & 4 & 0,26 \\
\hline \multicolumn{4}{|l|}{ DEBILIDADES } \\
\hline D1 Dificultades para la tecnificación masiva en la producción apícola. & 0,06 & 1 & 0,06 \\
\hline D2 No existe un plan definido para la distribución de productos apícolas. & 0,10 & 1 & 0,10 \\
\hline D3 Ausencia de estructura de costos y gastos para la producción apícola. & 0,06 & 1 & 0,06 \\
\hline D4 Poca variabilidad de consumidores frente a la venta de miel de abeja. & 0,10 & 2 & 0,19 \\
\hline $\begin{array}{l}\text { D5 Los productores pequeños obtienen menores precios de venta por } \\
\text { ineficientes procesos de negociación. }\end{array}$ & 0,10 & 2 & 0,19 \\
\hline $\begin{array}{l}\text { D6 Ausencia de apoyo por parte del gobierno para facilitar proyectos } \\
\text { identificables que favorezcan la comercialización. }\end{array}$ & 0,06 & 1 & 0,06 \\
\hline TOTAL - MATRIZ FACTORES INTERNOS EFI & 1,00 & & 2,68 \\
\hline \multicolumn{4}{|l|}{ OPORTUNIDADES } \\
\hline O1 Posibilidad de obtener una marca que se posicione en el mercado. & 0,04 & 4 & 0,14 \\
\hline $\mathrm{O} 2$ Crecimiento de la demanda interna por productos naturales. & 0,11 & 4 & 0,43 \\
\hline $\begin{array}{l}\text { O3 Corredor Económico en proyecto Olmos generará un incremento en } \\
\text { ventas ecológicas. }\end{array}$ & 0,11 & 3 & 0,32 \\
\hline O4 El FEN (Fenómeno del Niño) aumenta la oferta florística. & 0,11 & 4 & 0,43 \\
\hline $\begin{array}{l}\text { O5 Posibilidad de aporte financiero por entidades gubernamentales y privadas } \\
\text { interesadas en productos agrarios. }\end{array}$ & 0,07 & 3 & 0,21 \\
\hline O6 Interés de universidades públicas para hacer investigación en la zona. & 0,07 & 3 & 0,21 \\
\hline \multicolumn{4}{|l|}{ AMENAZAS } \\
\hline A1 Condiciones climáticas no permitan adecuada floración del bosque. & 0,07 & 4 & 0,29 \\
\hline A2 Adulteración de miel de abeja en mercados locales y/o regionales. & 0,11 & 4 & 0,43 \\
\hline A3 Competencia frente a una inestabilidad de precios de venta. & 0,07 & 3 & 0,21 \\
\hline A4 Peligro de cultivos transgénicos. & 0,11 & 3 & 0,32 \\
\hline A5 Peligro por la aparición de nuevas enfermedades en las abejas. & 0,07 & 3 & 0,21 \\
\hline A6 No ejecutar nuevos proyectos de inversión e investigación. & 0,07 & 2 & 0,14 \\
\hline TOTAL - MATRIZ EFE & 1,00 & & 3,36 \\
\hline
\end{tabular}

Las cantidades adquiridas en Chiclayo son $1 \mathrm{~kg}$ para $60 \%, 1 / 2 \mathrm{~kg}$ en $14 \%$ de casos, $9 \%$ compra $1 / 4 \mathrm{~kg}, 8 \%$ compra $2 \mathrm{~kg}$ y $9 \%$ otras cantidades. Las presentaciones preferidas son frascos de vidrio para $45 \%$; bolsa con etiqueta de procedencia, marca y peso en $39 \%$; frasco de plástico en $9 \%$; envases descartables en $6 \%$ y bolsa sin etiqueta en 1\%. En Lima, el 57\% acostumbra comprar botellas de $1 / 2 \mathrm{~kg}$, un $24 \%$ de $1 / 4$ $\mathrm{kg}, 8 \%$ de $1 \mathrm{~kg}$ y $11 \%$ otras cantidades. En las presentaciones son frascos de plástico
(71\%), vidrio (25\%) y granel (4\%). Para $41 \%$ de encuestados la miel de abeja es sustituto de azúcares y edulcorantes, complemento de alimentos $21 \%$ y suple a fármacos $38 \%$.

En Chiclayo las familias consideran a la miel de abeja como un insumo de su canasta básica resaltándola como sustituto del azúcar por $75 \%$ de encuestados y complemento de otros alimentos en $25 \%$. El $54 \%$ de familias destinan para su alimentación menos de 400 soles, el 39\% utilizan entre 401 a 800 soles y el 7\% asigna entre 801 a 1200 soles. 
En Lima el $10 \%$ de familias destinan a la alimentación menos de 400 soles, $31 \%$ de 401 a 800 soles y $59 \%$ entre 801 a 1200 soles, valores diferentes entre Chiclayo y Lima.

En Chiclayo los atributos de una buena miel de abeja es la consistencia y para reducir el riesgo de ser adulterada, el sabor pero que es difícil describir debido a la procedencia de diferentes fuentes florales, el color que se relaciona con el proceso y la fuente floral, variando desde transparente, ámbar, claro y caramelo, además que no se solidifique, los lugares de compra son los mercados aledaños (Tabla 9). En Lima, para reconocer la calidad se prioriza la consistencia $(30 \%)$ el color $(26 \%)$ y la marca $(20 \%)$, difiriendo de Chiclayo en que se prioriza el sabor a la marca. Los lugares donde se adquiere la miel son las tiendas naturistas por su garantía, le ofrecen las casas distribuidores de confianza, entre otros (Tabla 9).
Los consumidores de Chiclayo reconocen las marcas La Abejita 36\%, Olmos del Sur 25\%, Oxapampa 14\%, Toyva $8 \%$, Santa Natura $1 \%$, otras marcas $11 \%$ y no conocen $6 \%$. La marca Olmos del Sur proviene del distrito de Olmos que tiene como especies florales el algarrobo, zapote y espino, la marca "La Abejita" proviene de áreas de Chiclayo donde prima el algarrobo. El 75\% desconoce la procedencia de producción y el $25 \%$ lo conocen porque adquieren directamente del productor o de establecimientos donde son informados de su procedencia. En el mercado limeño se reconoce la marca Santa Natura 37\%, Oxapampa $31 \%$, Toyva $2 \%$, y diversas marcas o no conocen 30\%. En Lima el 84\% desconocen el lugar de procedencia de la miel de abeja, siendo que en Chiclayo existe mayor información pues esa ciudad es uno de los principales eslabones de distribución apícola y con mayor cercanía a las zonas productoras.

Tabla 8: Matriz del análisis de direccionamiento estratégico del primer cuadrante de crecimiento y desarrollo

\begin{tabular}{|c|c|c|}
\hline & FORTALEZAS & DEBILIDADES \\
\hline \multirow{6}{*}{ 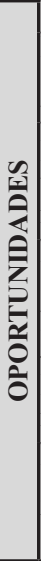 } & ESTRATEGIAS OFENSIVAS & ESTRATEGIAS ADAPTATIVAS \\
\hline & $\begin{array}{l}\text { FO1: Identificar rutas de comercio para } \\
\text { aprovechar el incremento de la demanda y las } \\
\text { ventas }(\mathrm{F} 1, \mathrm{O} 2, \mathrm{O} 3) \text {. }\end{array}$ & $\begin{array}{l}\text { DO1: Contar con asesores en desarrollo } \\
\text { apícola para manejar situaciones como plagas y } \\
\text { enfermedades en la apicultura (D1, O4). }\end{array}$ \\
\hline & $\begin{array}{l}\text { FO2: La certificación de miel permitirá la } \\
\text { ventaja competitiva y un posicionamiento en el } \\
\text { mercado a través de una marca líder (F2, O1). }\end{array}$ & $\begin{array}{l}\text { DO2: Definir protocolos de reconocimiento } \\
\text { sobre estructuras de costos para identificar } \\
\text { inversiones y/o gastos necesarios para la } \\
\text { proyección de la producción apícola (D3, O5). }\end{array}$ \\
\hline & $\begin{array}{l}\text { FO3: El trabajo organizado fortalecerá la } \\
\text { actividad frente al incremento de la demanda } \\
\text { y aprovechar los materiales utilizados (F4, } \\
\text { O2). }\end{array}$ & $\begin{array}{l}\text { DO3: Elegir canales de comercio óptimos para } \\
\text { la distribución eficiente y rentable (D2, O3). }\end{array}$ \\
\hline & $\begin{array}{l}\text { FO4: Identificar zonas apícolas para propiciar } \\
\text { mayor inversiones }(\mathrm{F} 6, \mathrm{O} 5, \mathrm{O} 6) \text {. }\end{array}$ & $\begin{array}{l}\text { DO4: Capacitación en reconocimiento de } \\
\text { calidad al productor y consumidor, para captar } \\
\text { un público más variado. (D4, D6, O5). }\end{array}$ \\
\hline & $\begin{array}{l}\text { FO5: Investigaciones en mejoramiento } \\
\text { genético, físico-químico y organoléptico para } \\
\text { tener manejo óptimo en la producción (F5, } \\
\text { O4). }\end{array}$ & \\
\hline
\end{tabular}

Tabla 9: Atributos que consideran la calidad del bien y lugares de compra en Chiclayo y Lima

\begin{tabular}{lcclcc}
\hline Atributos & $\begin{array}{c}\text { Distritos de } \\
\text { Chiclayo (\%) }\end{array}$ & $\begin{array}{c}\text { Distritos de } \\
\text { Lima (\%) }\end{array}$ & Lugares de compra & $\begin{array}{c}\text { Chiclayo } \\
(\%)\end{array}$ & $\begin{array}{c}\text { Lima } \\
(\%)\end{array}$ \\
\hline Consistencia & 33 & 30 & Mercado municipal & 54 & 23 \\
Por el sabor & 32 & 9 & Supermercados & 19 & 2 \\
Por el color & 28 & 26 & Tiendas naturistas & 6 & 32 \\
Por la marca & 1 & 21 & Bodegas & 1 & 1 \\
No se azucara & 4 & 1 & Lo ofrecen en casa & 10 & 27 \\
Confianza en el & 2 & 13 & Directo del productor & 4 & 8 \\
vendedor & & & Otros & 6 & 8 \\
\hline
\end{tabular}


Los consumidores de Chiclayo reconocen las marcas La Abejita 36\%, Olmos del Sur 25\%, Oxapampa 14\%, Toyva $8 \%$, Santa Natura $1 \%$, otras marcas $11 \%$ y no conocen $6 \%$. La marca Olmos del Sur proviene del distrito de Olmos que tiene como especies florales el algarrobo, zapote y espino, la marca "La Abejita" proviene de áreas de Chiclayo donde prima el algarrobo. El 75\% desconoce la procedencia de producción y el $25 \%$ lo conocen porque adquieren directamente del productor o de establecimientos donde son informados de su procedencia. En el mercado limeño se reconoce la marca Santa Natura 37\%, Oxapampa $31 \%$, Toyva 2\%, y diversas marcas o no conocen 30\%. En Lima el $84 \%$ desconocen el lugar de procedencia de la miel de abeja, siendo que en Chiclayo existe mayor información pues esa ciudad es uno de los principales eslabones de distribución apícola y con mayor cercanía a las zonas productoras.

\section{Conclusiones}

Del estudio se concluye que, existen diferencias de gestión productiva y comercial en los productores organizados que elaboran miel orgánica respecto a los productores individuales de miel convencional.

En Tongorrape no existen relaciones contractuales entre productor y distribuidor, los canales de comercio de miel de abeja convencional son relativamente simples con bajo poder de negociación de los productores y predominan redes centralizadas orientadas al mercado local y regional, en tanto, en la miel orgánica predomina la distribución descentralizada con canales directos y circuitos cortos para su comercio en el mercado regional, extrarregional y nacional.

La actividad apícola puede apuntalar la mejora de ingresos de los pobladores rurales y promover la sostenibilidad del bosque seco. Debido a las diferencias en las preferencias y conocimiento de la miel de abeja entre los consumidores de Chiclayo y Lima Metropolitana.

Se recomenda involucrar al gobierno regional y local para fomentar la organización, la tecnificación, la calidad y valor agregado en los productores, fortalecer la cadena productiva apícola propiciando el comercio con contratos y circuitos cortos, además de proveer información a los consumidores de las cualidades y del reconocimiento del producto ofrecido desde el bosque seco.

\section{Agradecimiento}

Al Consejo de Universidades Flamencas de Bélgica (VLIR) y la Universidad Nacional Agraria La Molina, que auspiciaron el estudio, a los productores apícolas de la localidad de Tongorrape y directivos de ASPROBOS que facilitaron la información.

\section{Literatura citada}

Alarcón, J.; Ordinola, M. 2002. Mercadeo de productos agropecuarios: teoría y aplicaciones al caso peruano. $1^{\text {ra }}$ edición. A4 Impresores, Perú. 374 p.

APEIM [Asociación Peruana de Empresas de Investigación de Mercados]. 2014. Lima. Disponible en www.apeim.com. pe.

Beltrán, R. 2013. Citología básica de los meristenos radiculares de las semillas de Prosopis pallida (Humb \& Bonpl. ex Willd.) "algarrobo pálido" mediante la impregnación argéntica. Revista de la Facultad de Ciencias Biológicas 33 (1): $1-12$.

Braunstein, M. 2006. Portal apícola. Argentina.

Caldentey, P.; De Haro, T. 2004. Comercialización de productos agropecuarios. $5^{\text {ta }}$ edición. Madrid Agrícola Española: Mundi Prensa 2004. $354 \mathrm{p}$.

Caldentey, P.; Gómez, A. 1993. Economía de los mercados agrarios. Madrid: Universidad de Córdoba. Madrid MundiPrensa 1993. 218 p.

Castañeda, M. 2016. Estrategia de comunicación para el sistema regional de áreas de conservación de Lambayeque. Gobierno Regional de Lambayeque. Chiclayo.

CENAGRO [IV Censo Nacional Agropecuario] 2012. Instituto Nacional de Estadística e Informática 2012. Disponible en www.censos.inei.gob.pe/ cenagro/tabulados

Coscia, A. 1978. Comercialización de productos agropecuarios. Buenos Aires. Editorial Hemisferio Sur.

Costanza, R.; D’Arge, R.; De Groot, R.; 
Farberk, S.; Grasso, M.; Hannon, B.; Limburg, K.; Naeem, S.; O’Neill, R.; Paruelo, J.; Raskin, R.; Suttonkk, P.; Van Den Belt, M. 1997. The value of the world's ecosystem services and natural capital. Nature 387:253-260.

Cuentas, M. 2015. El uso del espacio natural para el desarrollo del territorio: los bosques secos de algarrobo para las comunidades rurales en Lambayeque, 1985-2015. Revista Investiga Territorios 2: 105-118.

De Groot, R.; Alkemade, R.; Braat, L.; Hein, L.; Willemen, L. 2010. Challenges in integrating the concept of ecosystem services and values in landscape planning, management and decision making. Ecological Complexity 7:260272.

Fisher, B.; Turner, R.; Morling, P. 2009. Defining and classifying ecosystem services for decision making. Ecological Economics 68(3):643-653.

Indacochea, A.; Bazán, C.; Castañeda, J.; Céspedes, S. Gonzáles, E.; Morales, P. 2001. Ayacucho competitivo: competitividad a través de la apicultura. Lima. CARE PERÚ - USAID CETRUM PUCP.

INEI [Instituto Nacional de Estadística e Informática]. 2016. Censos Nacionales 2007, XI de población y VI de vivienda. Sistema de consulta de datos censales. Disponible en http://censos.inei.gob.pe/ cpv2007/tabulados/

INRENA [Instituto Nacional de Recursos Naturales]. 2001. Proyecto Algarrobo. Experiencias en el manejo participativo de los bosques secos del norte del Perú. Piura.

IPSOS [APOYO Opinión y Mercado S. A.]. 2013. Perfiles socioeconómicos. Lima. Disponible en www.ipsos.pe/Perfiles socioenconomicos 2013

Martínez, F. 2005. Comercialización agropecuaria: un enfoque económico de las estrategias comerciales. $1^{\text {ra }}$ edición. Santiago de Chile: Pontificia Universidad Católica de Chile 2005. 483 p.

MEA [Millennium Ecosystem Assessment, US]. 2005. Ecosystems and Human Well-Being: Biodiversity Synthesis. Washington, USA. World Resources Institute. $155 \mathrm{p}$.
Mendoza, G. 1991. Compendio de mercadeo de productos agropecuarios. $2^{\text {da }}$ edición. San José, Costa Rica. IICA. 343 p.

Philippe, J. 1990. Guía del apicultor. Madrid. Ediciones Mundi Prensa, 376 p.

Rodríguez, G.; Curetti, G.; Garegnani, G.; Grilli, G.; Partorella, F.; Paletto, A. 2016. La valoración de los servicios ecosistémicos en los ecosistemas forestales: un caso de estudio en Los Alpes Italianos. Revista Bosque 37(1): 41-52.

Sabogal, A. 2011. Estudio de la vegetación y el pastoreo en los bosques secos del norte del Perú con énfasis en la distribución de Ipomoea carnea Jacq. Sociedad Geográfica de Lima. 192 p.

SERFOR [Servicio Nacional Forestal y de Fauna Silvestre]. 2015. El análisis de servicios eco sistémicos forestales como herramienta para la formulación de políticas nacionales en el Perú. Lima.

Stern, L. 1999. Canales de comercialización. $5^{\text {ta }}$ edición. Madrid. Prentice Hall.

UICN [Unión Internacional para la Conservación de la Naturaleza]. 2015. Diagnóstico, identificación y valoración económica de servicios eco sistémicos, municipios de San Juan Nepomuceno y Santa Rosa de Cauca. Parques Nacionales Naturales de Colombia. Bogotá. 23 p.

Van der Heyden, D.; Camacho, P. 2006. Guía metodológica para el análisis de cadenas productivas. $2^{\text {da }}$ edición. Quito. RURALTER.

Yachi, K. 2014. Estudio socioeconómico de línea base del caserio El Choloque, CC. Tongorrape, Lambayeque. Lima. Universidad Nacional Agraria La Molina.

\section{(Footnotes)}

1 En el canal directo se identifican, Tipo 1: relación directa entre productor-consumidor; y Tipo 2: relación existente entre productor-intermediario-consumidor. El canal Tipo 2 sólo cuenta con un intermediario.

2 Canal indirecto con relación productorintermediario-consumidor en número de intermediarios mayor a 1 . 